This document is the accepted manuscript version of the following article:

Pastorek, J., Fencl, M., Rieckermann, J., \& Bares, V. (2021). Precipitation estimates from commercial microwave links: practical approaches to wet-antenna correction. IEEE Transactions on Geoscience and Remote Sensing. https://doi.org/10.1109/TGRS.2021.3110004

\title{
Precipitation Estimates from Commercial Microwave Links: Practical Approaches to Wet-antenna Correction
}

\author{
Jaroslav Pastorek, Martin Fencl, Jörg Rieckermann, and Vojtěch Bareš
}

\begin{abstract}
An inadequate correction for wet antenna attenuation (WAA) often causes a notable bias in quantitative precipitation estimates (QPEs) from commercial microwave links (CMLs) limiting the usability of these rainfall data in hydrological applications. This paper analyzes how WAA can be corrected without dedicated rainfall monitoring for a set of 16 CMLs. Using data collected over 53 rainfall events, the performance of six empirical WAA models was studied, both when calibrated to rainfall observations from a permanent municipal rain gauge network and when using model parameters from the literature. The transferability of WAA model parameters among CMLs of various characteristics has also been addressed. The results show that high-quality QPEs with a bias below 5\% and RMSE of $1 \mathrm{~mm} / \mathrm{h}$ in the median could be retrieved, even from sub-kilometer CMLs where WAA is relatively large compared to raindrop attenuation. Models in which WAA is proportional to rainfall intensity provide better WAA estimates than constant and timedependent models. It is also shown that the parameters of models deriving WAA explicitly from rainfall intensity are independent of CML frequency and path length and, thus, transferable to other locations with CMLs of similar antenna properties.
\end{abstract}

Index Terms - Attenuation, bias, commercial microwave links, rainfall, wet antenna.

\section{INTRODUCTION}

$\mathrm{R}$ AINDROPS cause considerable attenuation of a radio signal at millimeter wavelengths. Commercial microwave links (CMLs) routinely monitor transceived signal levels. The difference between the transmitted and received levels, TRSL [dB], can be used to derive quantitative precipitation estimates (QPEs) [1]. However, attenuation from raindrops along the CML path $A_{r}[\mathrm{~dB}]$ must be separated from other components of TRSL such as wet antenna attenuation (WAA) using the relation

$$
\begin{gathered}
T R S L=B+A \\
A=A_{w a}+A_{r}
\end{gathered}
$$

where $B[\mathrm{~dB}]$ represents baseline attenuation consisting of, e.g., free space loss and gaseous attenuation, $A[\mathrm{~dB}]$ stands for

This study was supported by the Czech Science Foundation under the project no. 20-14151J and by the Grant Agency of the Czech Technical University in Prague under the projects no. SGS19/045/OHK1/1T/11 and SGS20/050/OHK1/1T/11. observed attenuation after baseline separation (henceforth referred to as "observed attenuation"), and $A_{w a}[\mathrm{~dB}]$ represents WAA. Inadequate correction for WAA often causes substantial bias in CML QPEs [2] limiting their usability in hydrological applications such as the prediction of rainfall-driven runoff in urban areas [3].

To date, there is no unified approach to estimate WAA and reported WAA models follow different assumptions. For example, drying times of up to several hours have been reported [4], whereas other studies have not considered any wetting or drying dynamics at all, relating WAA only to rainfall intensity [5], [6]. It has also been suggested to estimate WAA based on water quantity and distribution (droplets, rivulets, water film) on antenna radomes [7], [8]. Recently, it has been shown that WAA can be estimated using antenna reflectivity acting as a proxy variable for water film thickness [9]. However, applying this model is significantly limited by the unavailability of the required antenna reflectivity measurements. Therefore, the complexity of the antenna (radome) wetting process, namely its dependence on antenna hardware properties (e.g., coating [10]) and on atmospheric conditions other than precipitation, remains a major challenge to reliable WAA estimation. It also negatively affects the transferability of WAA models among different CMLs and, thus, optimal WAA models should ideally be determined for each individual CML. This is especially true for models whose parameters depend on CML path length, e.g., [6]. However, optimal WAA model identification (e.g., for calibration purposes) on the level of individual CMLs is challenging for real-world application with networks consisting of a high number of CMLs. As noted by [11], maintenance of dedicated equipment for the retrieval of the needed reference rainfall observations is impractical for such networks. Consequently, application-focused studies with city or regional-scale CML networks have often not applied any WAA correction at all [12], [13] or have used only a simple constant offset model [3], [14], [15], [16]. Although the latter approach may be a reasonable choice when only 15-min TRSL maxima and minima are available [2], it can introduce considerable bias

J. Pastorek (e-mail: jaroslav.pastorek@fsv.cvut.cz), M. Fencl, and V. Bareš are with the Department of Hydraulics and Hydrology, Czech Technical University in Prague, 16627 Prague 6, Czech Republic.

J. Rieckermann is with the Department of Urban Water Management, Eawag: Swiss Federal Institute of Aquatic Science and Technology, Überlandstrasse 133, 8600 Dübendorf, Switzerland. 
in the resulting CML QPEs [3], [17]. To avoid such errors, Graf et al. [18] recently tested a time-dependent [4] and a semiempirical WAA model assuming a homogeneous water film on antenna radomes which depends on rain rate through a power law [7]. However, in the case of both WAA models, only a single set of fixed parameters for all of around 4000 CMLs from their extensive dataset was used and this did not address the suitability of the WAA model parameters for individual CMLs.

This study analyzes, for the first time, six empirical WAA models, including a newly proposed one, based on considerably different assumptions and tests their performance in detail. In contrast to previous studies, often limited by a low number of CMLs investigated [4], [7], [10], short time series of a few months [7], [14], [15] or 15-min CML data sampling intervals [14], [19], a rich dataset of more than two years of data retrieved from 16 CMLs with a sub-minute sampling rate was used. Motivated by the vision of reducing the costs of future studies with high numbers of CMLs, we also address the previously recognized need [11], [18] to minimize the amount of auxiliary data necessary for WAA estimation without compromising the quality of retrieved QPEs and, thus, we introduce three conceptual innovations not previously presented in relevant literature. Firstly, we show how the investigated empirical WAA models can be calibrated while notably minimizing the requirements on the reference rainfall data necessary, i.e., using only a rain gauge network with a spatial resolution of one gauge per $20-25 \mathrm{~km}^{2}$ and a temporal resolution of 15 minutes. Secondly, we analyze the variability in WAA model parameters optimized for different CMLs, thus indirectly assessing parameter uncertainties, and investigate which of the studied models can provide reliable WAA estimates without being calibrated for each individual CML. This includes a reformulation of a previously reported WAA model [5]. Thirdly, we suggest a procedure enabling the application of rainfall-dependent WAA models without any auxiliary rainfall observations, i.e., using only CML data.

\section{MATERIAL}

The data set originates from a case study in the Letñany suburb of Prague, Czech Republic [3]. Signal-level data from 16 CMLs are used, as well as rainfall observations from three tipping-bucket rain gauges (MR3, Meteoservis) from a permanent monitoring network operated by the municipal sewer authority with a density of one gauge per $20-25 \mathrm{~km}^{2}$ (Fig. 1). The CMLs (Mini-Link, Ericsson) belong to a major telecom operator, broadcast at $25-39 \mathrm{GHz}$ frequencies and feature path lengths between 816 and $5795 \mathrm{~m}$ (Table I). CML data were retrieved at a 10-s resolution with a common quantization of 1 $\mathrm{dB}$ and $0.33 \mathrm{~dB}$ for the transmitted and received signal power. Rain gauge data were recorded at a 1-min resolution. The dataset was collected between July 2014 and October 2016, excluding winter months (December-March) as CML signal attenuation by frozen precipitation, occurring in winter periods, is considerably different than that of liquid precipitation. Moreover, our monitoring setup is designed for periods with liquid precipitation only, as the rain gauges are not heated.
TABLE I

CHARACTERISTICS OF THE CMLS USED. FREQA AND FREQB ARE CML FREQUENCIES FOR THE TWO DIRECTIONS. THE NA VALUES INDICATE THAT RECORDS ARE NOT AVAILABLE.

\begin{tabular}{rrrr}
\hline \hline$\#$ & FreqA [GHz] & FreqB [GHz] & Path Length [m] \\
\hline 3 & $\mathrm{NA}$ & 32.63 & 816 \\
4 & 38.88 & 38.60 & 911 \\
5 & 24.55 & 25.56 & 1022 \\
6 & 37.62 & 37.62 & 1086 \\
7 & 37.62 & 38.88 & 1396 \\
8 & 37.62 & 38.88 & 1584 \\
9 & 31.82 & 32.63 & 1858 \\
11 & 38.88 & $\mathrm{NA}$ & 1979 \\
12 & 31.82 & 32.63 & 2611 \\
13 & 24.55 & 25.56 & 2957 \\
14 & 24.55 & 25.56 & 3000 \\
15 & $\mathrm{NA}$ & 25.56 & 3195 \\
16 & 24.55 & 25.56 & 3432 \\
17 & 25.56 & 24.55 & 4253 \\
18 & 24.55 & 25.56 & 4523 \\
19 & 24.55 & 25.56 & 5795 \\
\hline \hline
\end{tabular}

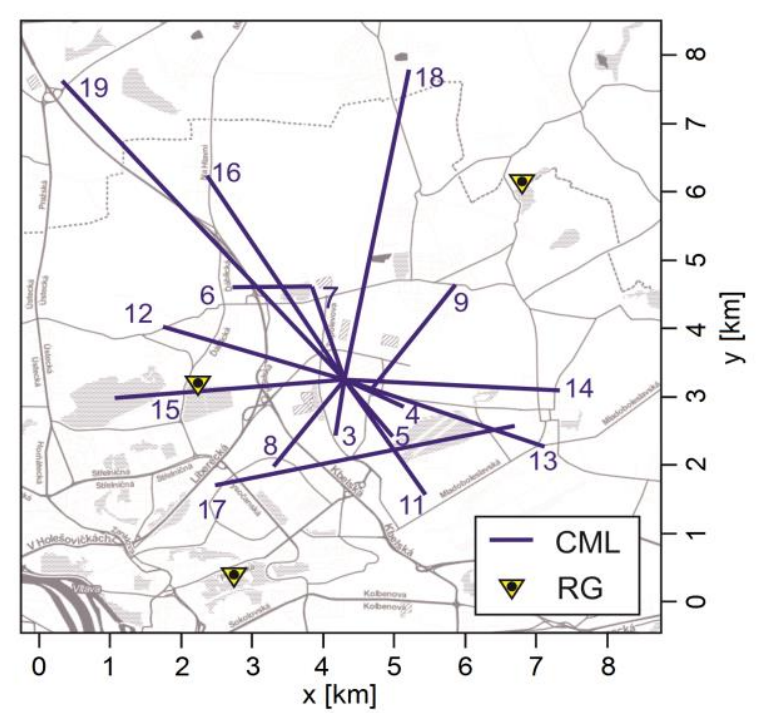

Fig. 1. Spatial relations of the CMLs and rain gauges (RG). CMLs are labeled with IDs (\# from Table I) which increase with CML path length.

\section{METHODS}

Attenuation data from the CMLs are processed (III.A) and corrected for WAA using six empirical WAA models (III.B). The resulting CML QPEs are evaluated against the rain gauge data from the municipal network (III.C).

\section{A. From signal levels to CML QPEs}

CML data processing steps before baseline separation, including a quality check and aggregation to a 1-min resolution, are done in the same way as in [3]. $B$ is assumed to equal TRSL (1) during dry periods. During wet periods, $B$ is estimated by linearly interpolating from the dry periods. Data available from both CMLs and rain gauges are used for the wet period identification. First, we identify wet timesteps for the CML data (mean TRSL of all CMLs) using a climatological threshold [20] defined as the 90th percentile of the rolling standard deviation of a 60-minute window. For the rain gauge data, timesteps are identified as wet when gauge tipping is observed at one or more gauges. Subsequently, wet periods are defined for both sensor types by setting the start of a wet period to one minute before 
the first observed wet timestep and the end to 60 minutes after the last one to ensure that baseline interpolation is not affected by wet antennas. Afterwards, the wet periods defined by the two sensor types are merged by taking the earliest starts and the latest ends. These periods are then used for the baseline separation using the linear baseline model.

After the baseline separation, $A_{w a}$ is subtracted (details in the next section) to obtain raindrop attenuation $A_{r}[\mathrm{~dB}]$. Then, $A_{r}$ is divided by the CML path length and thus transformed into specific raindrop attenuation $\gamma[\mathrm{dB} / \mathrm{km}]$ from which rainfall intensity $R[\mathrm{~mm} / \mathrm{h}]$ is calculated using the power-law relation

$$
R=\alpha \gamma^{\beta}
$$

with parameters $\alpha$ and $\beta$ according to ITU-R [21]. These parameter values are in very good agreement with values derived directly from drop size distribution observations [2], [5], however, they may not be optimal for other rain type regions [19].

\section{B. Empirical WAA models}

We evaluate six empirical models for WAA correction and a scenario without correcting for WAA (Zero) (Table II). For all models, it is assumed that WAA is estimated for two antennas, i.e., at both CML ends. The simplest approach is to model WAA $A_{w a}$ as a constant offset (O) [14]. In a more complex method, we model $A_{w a}$ as time-dependent, exponentially increasing towards an upper limit during wet periods, and decreasing exponentially afterwards (S) [4].

Next, we evaluate models where $A_{w a}$ depends on $R$. Valtr et al. [5] proposed a model (V) where the dependence on $R$ is explicit through a power law:

$$
A_{w a}=2 k^{\prime} R^{\alpha \prime}
$$

where $k^{\prime}$ and $\alpha^{\prime}$ are the power law parameters. We also analyze a model (KR) suggested by Kharadly and Ross [6] deriving $A_{w a}$ from observed attenuation $A$, i.e., depending on $R$ implicitly. However, as $A$ is dependent on CML path length, optimal parameters of the KR model would differ for two CMLs with the same hardware but with different path lengths. To eliminate this feature, we propose a model (KR-alt) in which $A_{w a}$ is bounded by an upper limit, as in [6], but derived from $R$ explicitly through a power law:

$$
A_{w a}=C\left(1-\exp \left(-d R^{z}\right)\right)
$$

where $C[\mathrm{~dB}]$ represents the maximal $A_{w a}$ possible, and $d$ and $z$ are power law parameters. Nevertheless, as optimal $C$ and $d$ values are not independent and can compensate for each other (similar to the KR model, see Fig. 5), we reduce the number of parameters to two by setting $d=0.1$.

WAA models with parameters independent of CML path length can also be formulated when $A_{w a}$ is derived explicitly from $\gamma$, not only $R$. However, it is unclear which of the two alternatives would provide WAA model parameters independent of CML frequency. Unlike $\gamma, R$ is independent of
CML frequency. The results from [7] suggest that $A_{w a}$ is also considerably less sensitive to CML frequency than $A_{r}$ or $\gamma$. Therefore, parameters of the models deriving $A_{w a}$ from $R$ explicitly are probably more transferrable among frequencies. To confirm this hypothesis, we reformulate the model of Valtr et al. [5] (V-alt), and replace $R$ with $\gamma$ so that:

$$
A_{w a}=2 p \gamma^{q}(6)
$$

where $p$ and $q$ are the power law parameters.

As neither $R$ nor $\gamma$ can be observed directly using CMLs, the $\mathrm{V}, \mathrm{V}$-alt, and KR-alt model equations must be rearranged to include only one unknown variable, $A_{w a}$, which can thus be quantified from $A$ (details in Appendix). The rearranged

\begin{tabular}{|c|c|c|}
\hline WAA model & $\begin{array}{l}\text { Parameter values from } \\
\text { the literature }\end{array}$ & Abbrev. \\
\hline $\begin{array}{l}\text { Zero WAA (no WAA } \\
\text { correction) }\end{array}$ & - & Zero \\
\hline Constant (non-zero) offset & $\begin{array}{l}A_{w a}=1.585 \mathrm{~dB} \\
\text { (the mean of the optimal } \\
\text { values identified in [14]) }\end{array}$ & $\mathrm{O}$ \\
\hline $\begin{array}{l}\text { Dynamic (time-dependent) } \\
\text { WAA (Schleiss et al. [4]) }\end{array}$ & $\begin{array}{l}W=2.3 \mathrm{~dB} \quad \tau_{w}=15 \mathrm{~min} \\
\text { (from [4]) }\end{array}$ & $S$ \\
\hline $\begin{array}{l}\text { Depending on } A \text { explicitly with } \\
\text { an upper limit } \\
\text { (Kharadly and Ross [6]) }\end{array}$ & $\begin{array}{l}C=8 \mathrm{~dB} \quad d=0.125 \\
\text { (from [6]; for } 27 \mathrm{GHz} \text { ) }\end{array}$ & $\mathrm{KR}$ \\
\hline $\begin{array}{l}\text { Depending on } R \text { explicitly with } \\
\text { an upper limit }\end{array}$ & - & KR-alt \\
\hline $\begin{array}{l}\text { Power-law relation to } R \\
\text { (Valtr et al. [5]) }\end{array}$ & $\begin{array}{l}k^{\prime}=0.68 \quad \alpha^{\prime}=0.34 \\
\text { (from [5]) }\end{array}$ & V \\
\hline $\begin{array}{l}\text { Power-law relation to } \gamma \\
\text { (reformulated Valtr et al. [5]) }\end{array}$ & 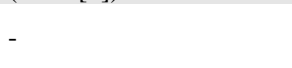 & V-alt \\
\hline
\end{tabular}
equations are then solved numerically.

TABLE II

OVERVIEW OF THE EMPIRICAL WAA MODELS AND THEIR PARAMETERS

\section{Calibration and performance of WAA models}

The WAA models studied are evaluated when using parameter values taken from the literature, if available, and when calibrated to rainfall data from the three municipal rain gauges. Moreover, for each WAA model, calibration is done in three scenarios: i) separately for each of the 16 CMLs; ii) separately for each frequency band; and iii) for all CMLs at once.

From the 3-year period, only hydrologically relevant rainfall events (total rainfall depth $H>2 \mathrm{~mm}$ ) are selected for calibration and evaluation. After eliminating events with substantial data gaps, 53 events (360 hours) are available, from which we randomly select 25 (281 hours) for WAA model calibration. Model parameters are optimized by comparing the CML QPEs with the mean $R$ of the three municipal rain gauges. Both data sets are aggregated from a 1-min to a 15-min resolution to reduce observation noise. The root mean square error $(R M S E)$ is used as the objective function and optimized with simulated annealing, an optimization method designed for complicated non-linear functions with many local minima [22]. For calibration scenarios using multiple CMLs at once, the mean RMSE of the CMLs is optimized.

Once the optimal WAA model parameters are identified, they are used to derive CML QPEs for the remaining 28 events (349 hours) not used for calibration. The CML QPEs are aggregated 
from the 1-min to the 15-min resolution and evaluated by direct comparison with the rain gauge data (the mean of the three gauges in the 15-min resolution). The QPEs are evaluated individually for each CML using a time series consisting of all 28 events, the performance for individual events is not quantified. Performance metrics employed are: i) the relative error of the rainfall depth $d H$ [\%] reflecting the bias; ii) the root mean square error $R M S E[\mathrm{~mm} / \mathrm{h}]$; and iii) the Spearman rank correlation coefficient SCC [-] which quantifies the strength of a monotonic relationship between two variables and is independent of both linear and non-linear bias.

\section{RESUlTS}

Firstly, the performance of the estimated CML QPEs summarized for all CMLs is presented. Secondly, the QPEs are investigated in closer detail on the level of individual CMLs.

The results summarized in Fig. 2 show that, when calibrated individually for each CML, models in which $A_{w a}$ is proportional to $R$ (KR, V, KR-alt, V-alt) can lead to CML QPEs with a bias lower than $5 \%$ in the median (up to $10 \%$ for most CMLs) and with $R M S E$ between 0.8 and $1.2 \mathrm{~mm} / \mathrm{h}$. Models explicitly relating $A_{w a}$ to $R$ (V and KR-alt) attain similarly good values (median bias less than 5\%, standard deviation 18\%) not only when calibrated individually for each CML, but also when calibrated for groups of CMLs with the same frequency and for all CMLs at the same time. Similarly, RMSE obtained using these two models is almost the same, between 0.8 and 1.2 $\mathrm{mm} / \mathrm{h}$, for all three calibration approaches. A very similar performance is reached using the $\mathrm{V}$-alt model, which relates $A_{w a}$ to $\gamma$, when calibrating separately for each of the three frequency bands. However, when calibrating the $\mathrm{V}$-alt model for all CMLs at once, the standard deviation of $d H$ increases to $25 \%$ and $R M S E$ values reach up to $1.4 \mathrm{~mm} / \mathrm{h}$ for some CMLs. Calibrating models $\mathrm{O}$ and $\mathrm{S}$ leads to markedly underestimated $d H$ values for most CMLs (around 40\% in median) for all three calibration approaches. This also affects the respective $R M S E$ values which are around $1.4 \mathrm{~mm} / \mathrm{h}$ in the median for all calibration approaches. Interestingly, using the $S$ model with parameter values from the literature leads to a lower bias for most CMLs ( $d H-20 \%$ in the median). However, the RMSE is virtually the same as for the calibrated model, with only a slightly larger variance. For other WAA models, the literature values perform, in general, worse than those optimized during the calibration, both in terms of $d H$ and RMSE. As expected, without the WAA correction (scenario Zero), CML QPEs are considerably overestimated (median $d H$ ca. 200\%, RMSE ca. $3 \mathrm{~mm} / \mathrm{h}$ ).

The correlation in terms of SCC (Fig. 2 bottom) reaches very similar values (about 0.85 in median) for all WAA models in which $A_{w a}$ is proportional to $R$ (KR, V, KR-alt, V-alt), regardless of whether/how they are calibrated. Only negligibly lower values are reached when not using any WAA model at all (scenario Zero). For most CMLs, SCC values between 0.8 and 0.85 are associated with the $\mathrm{O}$ and $\mathrm{S}$ models with parameter values from the literature. Calibrating these two models has led not only to a considerable underestimation of rainfall, but also to relatively low SCC values (medians between 0.65 and 0.76 ).

In addition, we analyze the estimated QPEs on the level of individual CMLs for two WAA modelling scenarios. First,
CML QPEs derived using the commonly used O model with parameter values from literature are compared with the rain gauge data (Fig. 3). Next, representing the better performing WAA models from above, the same is done for the $\mathrm{V}$ model with parameters optimized for all CMLs at once (Fig. 4). The V model leads to a distinct improvement over the $\mathrm{O}$ model. The $\mathrm{V}$ model reduces the bias for low and high $R$ and thus removes the dependence of errors in CML QPEs on $R$. Therefore, the performance metrics $d H$ and $R M S E$ are improved for most CMLs, however, the change of SCC is practically negligible. The reduction of errors is most significant for the shortest CMLs, as the relative contribution of $A_{w a}$ to $A$ decreases with the increasing path length.

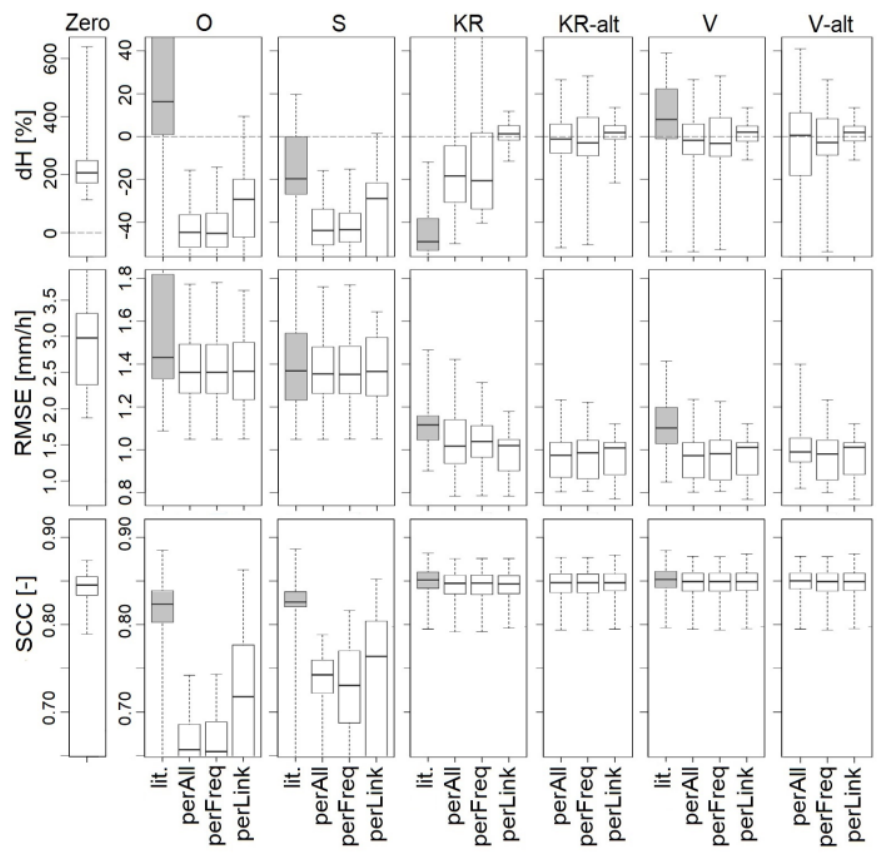

Fig. 2. Boxplots showing variation in the performance of the QPEs from the 16 individual CMLs quantified by the performance metrics $d H$ (top), RMSE (middle), and SCC (bottom). CML QPEs have been derived without WAA correction (Zero) and using the six WAA models. Sub-boxplots show the effect of WAA model calibration (lit. - parameter values from literature, perAll calibrated for all CMLs at once, perFreq - calibrated separately for CMLs operating at the three various frequency bands, perLink - calibrated separately for each CML). Note the different ranges of the y-axes for the Zero model.

We also present parameter values used for the WAA model evaluation, both optimized during calibration and taken from the literature (Fig. 5). Optimized parameter values of the V and KR-alt models are similarly located in their parameter spaces. Moreover, optimal parameter values for the three various frequency bands are, for these two models, located very close to the optimal values obtained when calibrating for all CMLs at once. This stands in contrast to the V-alt method for which a dependence between the frequency band and the optimal parameter values can be seen. For the KR model, the clear dependence of the two model parameters is most striking. For the $\mathrm{S}$ model, optimal values of the $W$ parameter are similar to the parameter values of the $\mathrm{O}$ model. However, there is no clear relation to the CML frequency for either of these two WAA models. Parameter values taken from the literature are, in all four cases, located relatively close to the optimized parameters. 

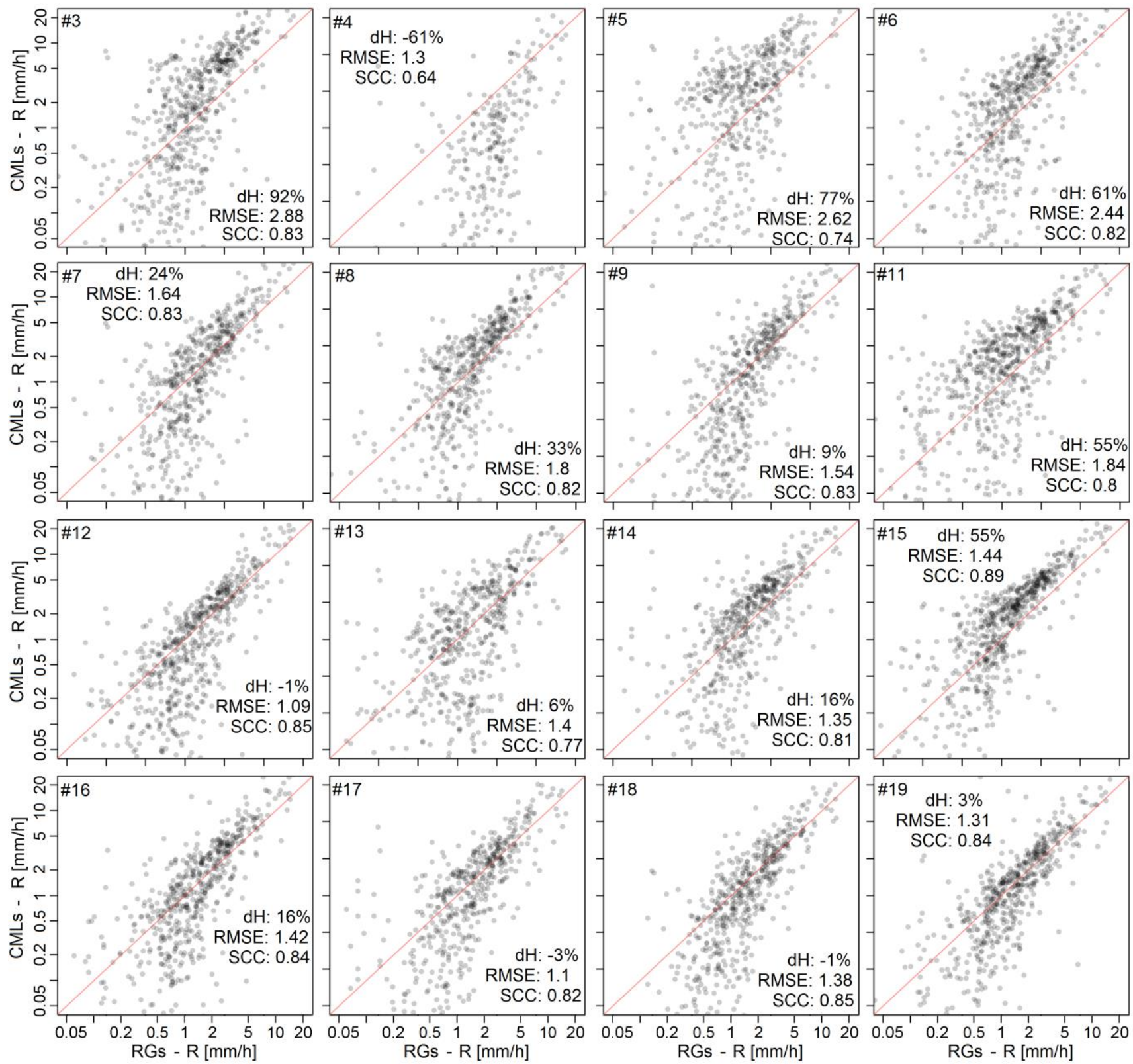

Fig. 3. Scatter plots comparing rain gauge data (RGs) with CML QPEs for the O model with parameters from the literature. Note that the axes are in logarithmic scales. The presented 15-minute data from the 28 rainfall events used for the evaluation represent 349 hours of observations. In 835 out of the 1,401 time steps, rain gauge data contain non-zero records. Most points with RG rainfall intensity below $0.3 \mathrm{~mm} / \mathrm{h}$ are out of the plotting range, as the respective CML QPEs are below $0.05 \mathrm{~mm} / \mathrm{h}$

\section{DISCUSSION}

The best results, in terms of $d H$ and $R M S E$, are, in general, achieved for the models in which WAA $A_{w a}$ is proportional to rainfall intensity $R$ (KR, V, KR-alt, V-alt). For these models, QPEs of the same high quality can be obtained when calibrating for each CML separately. However, the V model and the newly proposed KR-alt model, which both relate $A_{w a}$ to $R$ explicitly, perform very well, even when using the same parameter set for all CMLs. As the KR model relates $A_{w a}$ to $A$, which is dependent on CML path length, it performs markedly worse when using the same parameter set for more CMLs. The V-alt model performs very well when using the same parameters for CMLs operating at one frequency band and moderately worse when using the same parameter set for all frequency bands. This is in agreement with the calibrated model parameter values (Fig. 5) and supports the hypothesis that the parameters of models deriving $A_{w a}$ from $R$ explicitly (V, KR-alt) are more transferrable among CMLs of various frequencies than the parameters of models deriving $A_{w a}$ explicitly from $\gamma$ (V-alt).

The results of calibrating the $\mathrm{O}$ and $\mathrm{S}$ models resemble each other in terms of estimated rainfalls (Fig. 2), optimal model parameters (Fig. 5), and WAA levels (Fig. 6). The rainfall underestimation (i.e., WAA overestimation) associated with the $\mathrm{O}$ and $\mathrm{S}$ models is likely caused by different optimal parameter values for RMSE, used as the calibration objective function, and $d H$ due to the systematic errors in rainfall estimates when modelling WAA as completely or almost constant. 

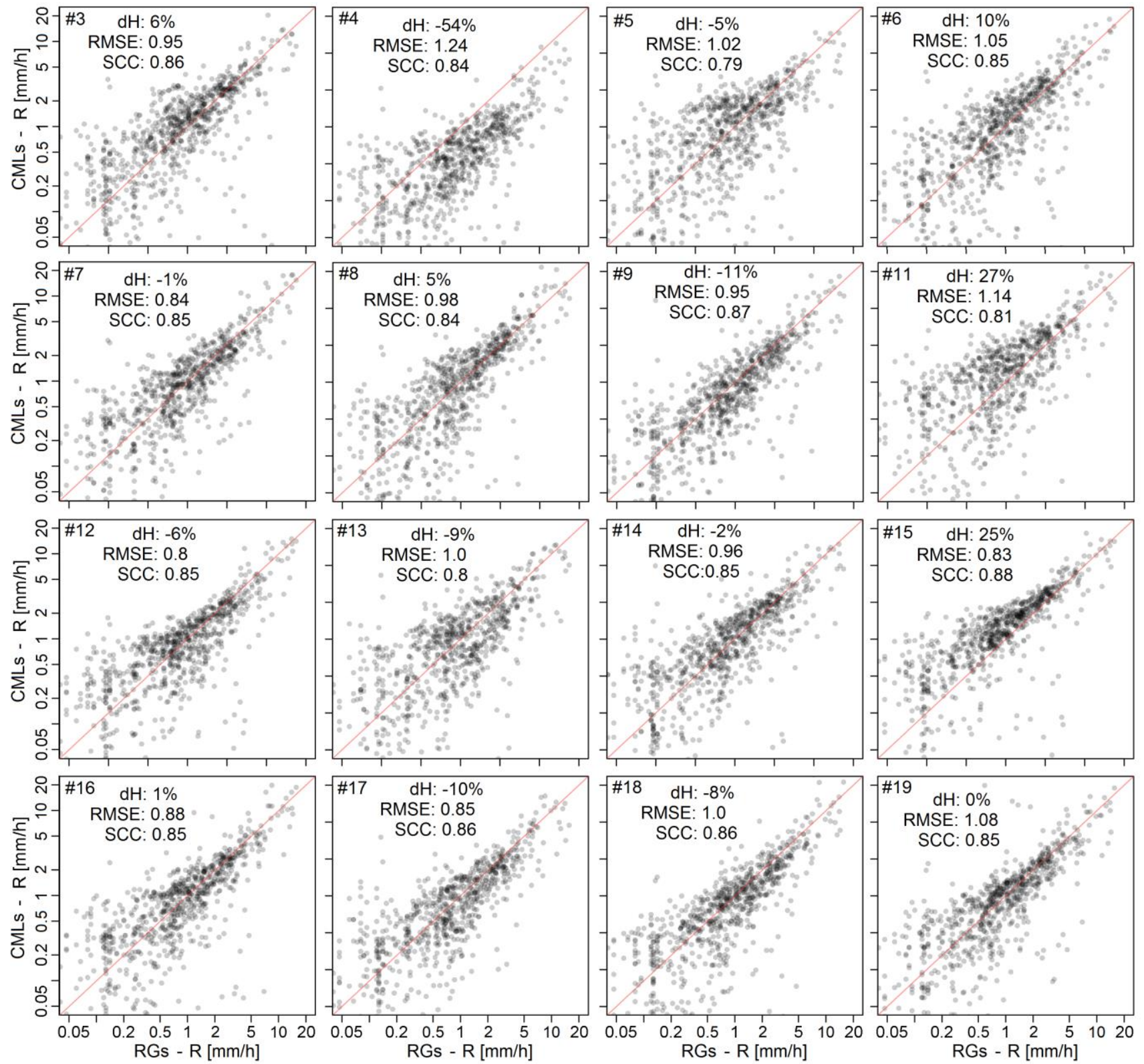

Fig. 4. Scatter plots comparing rain gauge data (RGs) with CML QPEs for the V model with the same parameters used for all the CMLs (obtained by optimizing for all CMLs at once). Note that the axes are in logarithmic scales. The presented 15 -minute data from the 28 rainfall events used for the evaluation represent 349 hours of observations. In 835 out of the 1,401 time steps, rain gauge data contain non-zero records.

In total, our results show that unbiased CML QPEs could be retrieved without the need for extensive additional rainfall monitoring when empirical models for WAA estimation are calibrated to rainfall data from the permanent municipal rain gauge network. Models in which WAA is dependent on rainfall intensity provide the best WAA estimates. Moreover, models explicitly relating WAA to rainfall intensity can provide optimal results even when using the same set of parameter values for CMLs of different characteristics.

The presented results confirm the importance of appropriately correcting for WAA when deriving QPEs from CMLs which is in agreement with previous research [2]. In particular, the results imply that modelling WAA as constant $(\mathrm{O}$ model) is not satisfying when TRSL data in 1-min resolution are available. This is in accordance with [3], [17] and contradicting [11], however, it should be noted that the latter study focused on WAA estimation for purposes of CML network design and investigated E-band CMLs. Nonetheless, our findings do not dispute the statement that this approach may be a reasonable choice if only 15-min TRSL maxima and minima are available [2].

It is shown that the most accurate rainfall estimates are associated with models relating WAA to rainfall intensity, which is in agreement with the WAA estimation approaches presented in [5], [6], [7], or [17]. On the other hand, having provided a comparison of the performance of different WAA models, Schleiss et al. [4] came to different conclusions. Although their results correspond to ours in terms of RMSE, not only for the scenario without WAA correction (Zero; 3.15 $\mathrm{mm} / \mathrm{h})$, but also for the WAA models $\mathrm{O}(1.34 \mathrm{~mm} / \mathrm{h})$ and $\mathrm{KR}$ 
$(0.91 \mathrm{~mm} / \mathrm{h})$, they observed the best performance for the timedependent $\mathrm{S}$ model $(0.72 \mathrm{~mm} / \mathrm{h})$. It should be noted that they used data from only a single CML and that the parameter values differed from those used in our study because the models were calibrated to local rainfall data from five disdrometers along the CML path. Since, in our case, the S model has only performed (and generally behaved) very similarly as the constant $\mathrm{O}$ model, it seems that, in accordance with [10], wetting dynamics play a much smaller role for the antennas used in this study than for those analyzed by Schleiss et al. [4]. Recently, similar behavior was observed by Graf et al. [18] who found that a semiempirical WAA model assuming a homogeneous water film on antenna radomes dependent on rain rate through a power law [7] led to more precise CML QPEs than the S model. As their analysis was based on a large country-wide dataset of around 4000 CMLs, it can be concluded that CML antennas for which WAA is not affected by the wetting dynamics are rather usual.

However, the relevance of our findings for other CML networks should be subject to further research. Firstly, it is likely that the capacity of rainfall data from rain gauge networks for calibrating WAA models will depend on gauge network density as the correlation among the gauges decreases with increasing distance. Aggregating the data to coarser resolutions for the calibration might improve the results as it would improve the correlation [23]. Nevertheless, if the sensors are too far from each other, it might be more appropriate to use longterm (e.g., monthly) precipitation heights.

Secondly, the reference areal rainfall used to evaluate the performance of the WAA models has been derived from the same three rain gauges that had been previously used to calibrate the WAA models. However, the rain gauge network density of one gauge per $20-25 \mathrm{~km}^{2}$ might not be sufficient to reliably represent areal rainfall for events with high spatial variability, e.g., storms with small convective cells. Therefore, out of the 28 individual rainfall events used for the WAA model evaluation, we have identified 11 events with the highest variability among the rain gauges and repeated our analysis using only the data from the remaining 17 events. Differences between the results for the 17 events and for all 28 events together are subtle. The estimated rainfall heights are slightly higher when evaluating all 28 events together than when using the 17 less variable events only. However, differences in terms of RMSE and SCC are minimal, and mutual relations of the individual WAA models and calibration scenarios are not affected.

Due to the use of only three rain gauges, we are also not able to precisely estimate rainfall starts and ends specifically for each CML. Therefore, the process of wet period identification has been designed to avoid classifying wet timesteps as dry, rather than vice versa. This approach makes wet periods longer, however, as the baseline is relatively stable [4], the order of errors in the estimated baseline levels is well below $1 \mathrm{~dB}$.

Next, all CMLs used in this study are from the same product family of the same manufacturer (Ericsson, Mini-Link) and have aged similarly due to exposure to similar climatic conditions. However, different behavior might be observed for CML antenna hardware of different producers, exposed to different climates for different time periods, or for other specific conditions (e.g., non-zero antenna elevation angles), and thus, the results of this study might not be directly applicable in such circumstances.
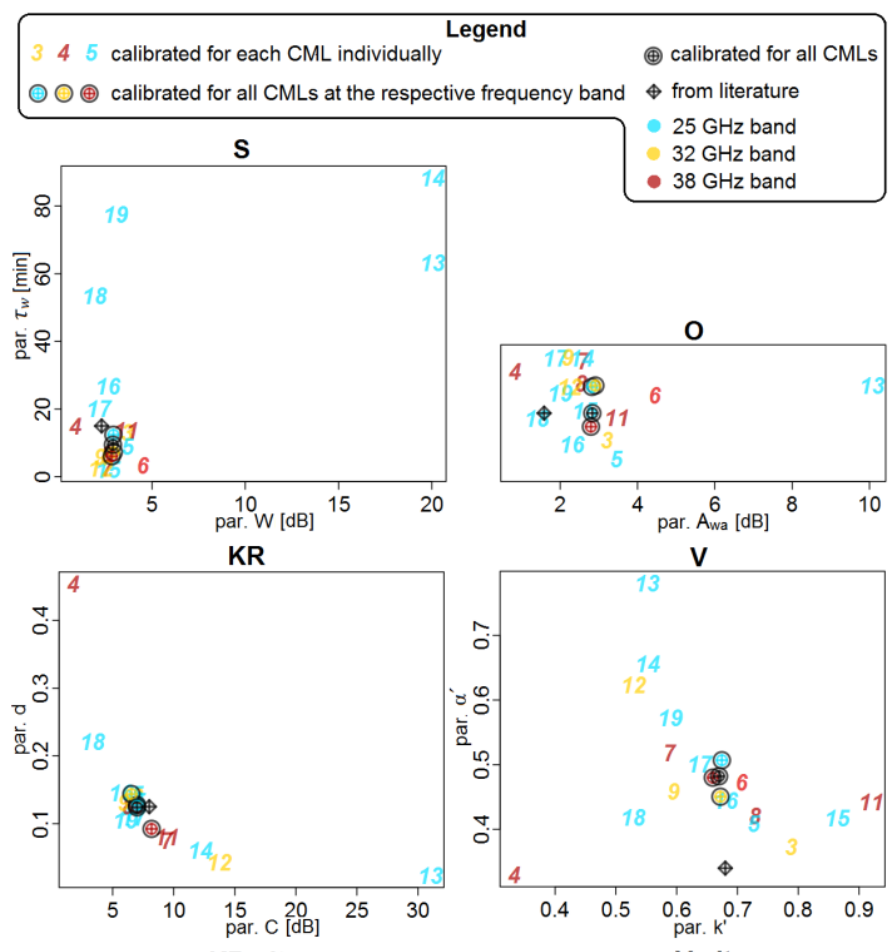

v
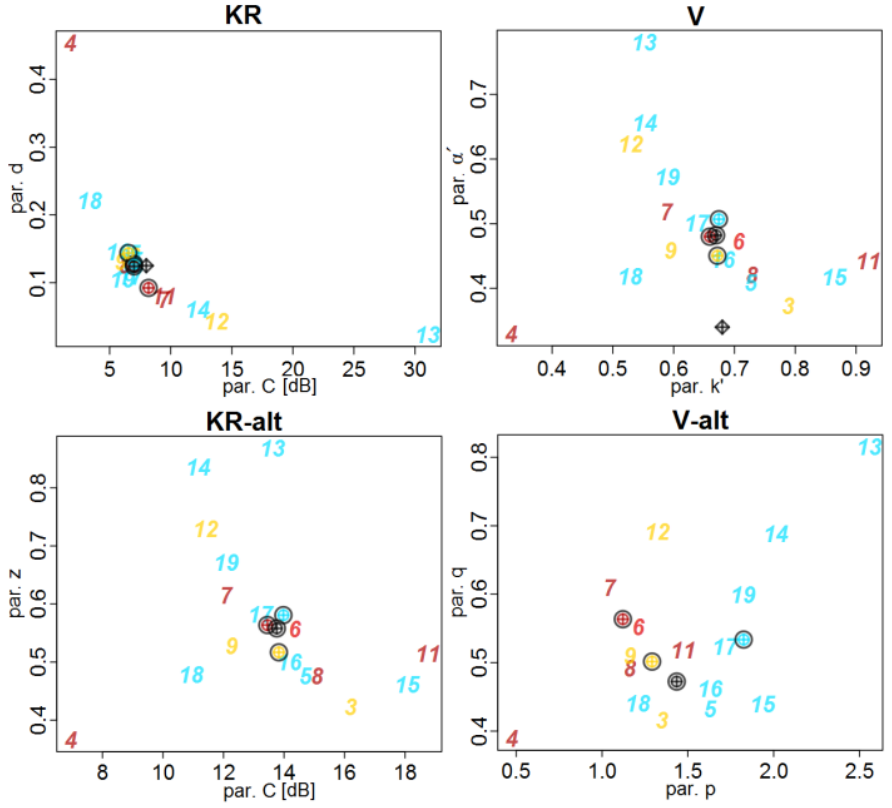

Fig. 5. WAA model parameter values used for WAA model evaluation, both optimized during calibration and taken from literature (if available). The numbers indicate CML IDs and the colors indicate frequency.
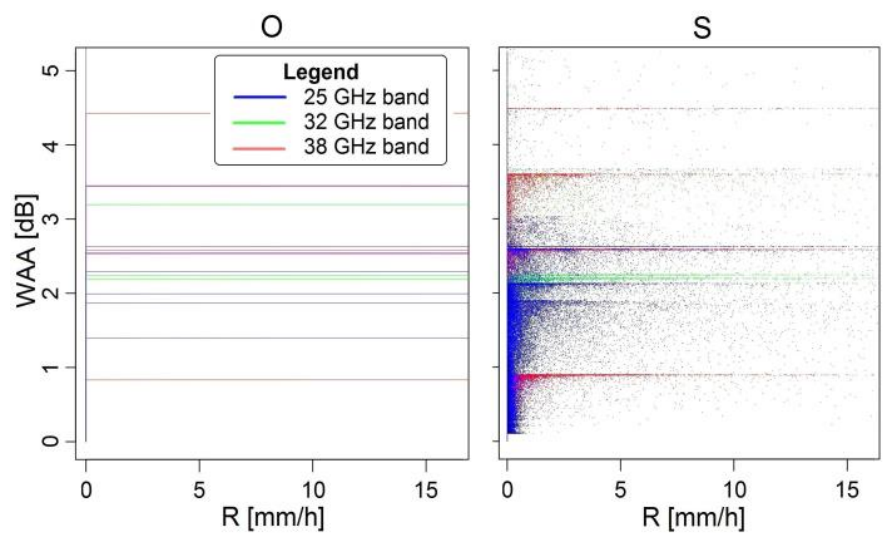

Fig. 6. WAA levels obtained using the $\mathrm{O}$ and $\mathrm{S}$ WAA models when calibrated separately for each CML in relation to the respective CML QPEs. The vertical line in the left panel at $R=0 \mathrm{~mm} / \mathrm{h}$ is caused by the nature of the $\mathrm{O}$ model. If observed attenuation $A$ is lower than a given parameter value of the $\mathrm{O}$ model, WAA is considered equal to $A$, i.e., there is no rainfall observed. 
Lastly, it should be noted that the $\mathrm{V}$ model was originally derived [5] by using one of our 16 CMLs and data from one of the three summer seasons that we have investigated herein.

\section{CONCLUSIONS}

We have shown in this study that virtually unbiased QPEs could be retrieved from CMLs without the need for dedicated rainfall monitoring campaigns. CML QPEs with a bias lower than $5 \%$ and $R M S E$ of $1 \mathrm{~mm} / \mathrm{h}$ in the median have been obtained when the empirical models for WAA estimation have been calibrated to rainfall data from the permanent municipal rain gauge network with a spatial resolution of one gauge per 20-25 $\mathrm{km}^{2}$. It has been shown that such high-quality QPEs can even be derived from short, sub-kilometer CMLs where WAA is relatively large compared to raindrop attenuation. Models relating WAA to rainfall intensity, implicitly or explicitly, have led to the best results. For the latter, parameter sets have been found to be suitable for CMLs of various path lengths operating at various frequency bands, which could thus be transferred to other locations with CMLs of similar antenna hardware characteristics. Moreover, it has been demonstrated how these WAA models can be successfully applied without any auxiliary rainfall observations, i.e., using CML data only.

This study has confirmed both the potential of CMLs as a source of high-quality rainfall data and the importance of appropriate WAA correction when deriving the QPEs. The presented advances in minimizing the requirements on auxiliary data necessary, both during the calibration of WAA models and during their implementation in the CML data processing routine, represent a legitimate step towards the retrieval of reliable QPEs from large CML networks in conditions where rainfall data are scarce. However, since the potential usefulness of CML QPEs increases with the decreasing availability of other rainfall (or other reference) data, further studies are needed, ideally with extensive datasets containing different CML hardware, to advance our capacity to correct for WAA in the data-scarce conditions. This would also be greatly beneficial for the application of CML QPEs in quantitative hydrological tasks such as urban rainfall-runoff predictions.

\section{APPENDIX}

This appendix shows how WAA models dependent on rainfall intensity can be used during the CML data processing routine without the need for auxiliary rainfall observations. In particular, we formulate single-unknown equation forms of the models which relate WAA $A_{w a}[\mathrm{~dB}]$ explicitly to rainfall intensity $R[\mathrm{~mm} / \mathrm{h}]$ and to specific raindrop attenuation $\gamma$ $[\mathrm{dB} / \mathrm{km}]$. Using these rearranged equations, $A_{w a}$ can be quantified directly from observed attenuation $A[\mathrm{~dB}]$, e.g., by solving the equations numerically. The relation between $\mathrm{V}$ and $\mathrm{V}$-alt models is also provided.

\section{$V$ model}

The following original equation of $\mathrm{V}$ model [5], where $k^{\prime}$ and $\alpha^{\prime}$ are the WAA model's power law parameters, requires independent observation of the rainfall intensity $R$ as its input:

$$
A_{w a}=2 k^{\prime} R^{\alpha \prime} .
$$

However, it holds that

$$
R=\alpha \gamma^{\beta}
$$

where $\alpha$ and $\beta$ are the power law parameters with values dependent on CML frequency and polarization. Moreover, if $L$ $[\mathrm{km}]$ is the CML path length, then

$$
\gamma=\left(A-A_{w a}\right) / L .
$$

Therefore, using (A.2) and (A.3), the original equation (A.1) can be rearranged into the following single-unknown form which can be used to quantify $A_{w a}$ from $A$ :

$$
A_{w a}=2 k^{\prime}\left(\alpha\left(\left(A-A_{w a}\right) / L\right)^{\beta}\right)^{\alpha \prime} .
$$

\section{$V$-alt model}

Starting from its original equation in which $A_{w a}$ is explicitly dependent on $R$ (A.1), the $\mathrm{V}$ model can be reformulated using the relation (A.2) in the following manner:

$$
A_{w a}=2 k^{\prime} R^{\alpha \prime}=2 k^{\prime}\left(\alpha \gamma^{\beta}\right)^{\alpha \prime}=2 k^{\prime} \alpha^{\alpha \prime} \gamma^{\beta \alpha \prime} .
$$

If $p=k^{\prime} \alpha^{\alpha \prime}$ and $q=\beta \alpha^{\prime}$, then the following equation, referenced to as $\mathrm{V}$-alt, represents a reformulation of the $\mathrm{V}$ model in which $A_{w a}$ is explicitly dependent on $\gamma$ :

$$
A_{w a}=2 p \gamma^{q}
$$

where $p$ and $q$ are power law parameters of the WAA model.

As with the original V model, the V-alt model (A.6) can also be rearranged using the relation (A.3) into a single-unknown form which can be used to quantify $A_{w a}$ from $A$ :

$$
A_{w a}=2 p\left(\left(A-A_{w a}\right) / L\right)^{q} .
$$

\section{KR-alt model}

The following original KR model [6], where $C$ [dB] and $d$ are the model parameters, relates $A_{w a}$ explicitly to $A$ :

$$
A_{w a}=C(1-\exp (-d A)) .
$$

However, as $A$ is affected by CML path length, optimal parameters of the KR model would differ for two CMLs with the same hardware but different path lengths. To eliminate this feature, we have proposed the following model (KR-alt) in which $A_{w a}$ is, similar to [6], bounded by an upper limit, but derived from $R$ explicitly through a power law:

$$
A_{w a}=C\left(1-\exp \left(-d R^{z}\right)\right)
$$

where $C, z$, and $d$ are parameters of the WAA model; $C$ [dB] represents the maximal $A_{w a}$ allowed; as $C$ and $d$ values are not independent and can compensate for each other, we reduce the 
number of parameters of this model to two by setting $d=0.1$.

Analogous to the $\mathrm{V}$ model, the KR model equation can also be rearranged by applying (A.2) and (A.3) into a singleunknown form which can be used to quantify $A_{w a}$ from $A$ :

$$
A_{w a}=C\left(1-\exp \left(-d\left(\alpha\left(\left(A-A_{w a}\right) / L\right)^{\beta}\right)^{z}\right)\right) \text {. }
$$

\section{ACKNOWLEDGMENT}

The authors would like to thank T-Mobile Czech Republic, a.s., for providing the CML data, and especially Pavel Kubík for assisting with their numerous requests. They would also like to thank Pražská vodohospodářská společnost, a.s., for providing rainfall data from their rain gauge network. They are grateful for the analytical help of Andreas Scheidegger from Eawag regarding numerical equation solving and the general methodology.

\section{AUTHOR CONTRIBUTIONS}

Jaroslav Pastorek (JP), with contributions from all other authors, wrote and structured the main text. Vojtěch Bareš (VB), with inputs from all other authors, framed the scope of the study. JP, with inputs from Martin Fencl (MF) and Jörg Rieckermann (JR), conceptualized the methodology. VB and MF managed the access to the measuring devices. VB, MF, and JP collected the data. MF and JP processed the data. JP ran the simulation experiments, statistically evaluated their results, and created the figures. VB and JR supervised the research. All authors reviewed and edited the paper.

\section{REFERENCES}

[1] D. Atlas and C. W. Ulbrich, "Path- and Area-Integrated Rainfall Measurement by Microwave Attenuation in the $1-3 \mathrm{~cm}$ Band," J. Appl. Meteorol., vol. 16, no. 12, pp. 1322-1331, Dec. 1977, doi: 10.1175/1520-0450(1977)016<1322:PAAIRM>2.0.CO;2.

[2] C. Chwala and H. Kunstmann, "Commercial microwave link networks for rainfall observation: Assessment of the current status and future challenges," WIREs Water, vol. 6, no. 2, p. e1337, Mar. 2019, doi: 10.1002/wat2.1337.

[3] J. Pastorek, M. Fencl, J. Rieckermann, and V. Bareš, "Commercial microwave links for urban drainage modelling: The effect of link characteristics and their position on runoff simulations," J. Environ. Manage., vol. 251, p. 109522, Dec. 2019, doi: 10.1016/j.jenvman.2019.109522.

[4] M. Schleiss, J. Rieckermann, and A. Berne, "Quantification and Modeling of Wet-Antenna Attenuation for Commercial Microwave Links," IEEE Geosci. Remote Sens. Lett., vol. 10, no. 5, pp. 1195-1199, 2013, doi: 10.1109/LGRS.2012.2236074.

[5] P. Valtr, M. Fencl, and V. Bareš, "Excess Attenuation Caused by Antenna Wetting of Terrestrial Microwave Links at $32 \mathrm{GHz}$," IEEE Antennas Wirel. Propag. Lett., vol. 18, no. 8, pp. 1636-1640, Aug. 2019, doi: 10.1109/LAWP.2019.2925455.

[6] M. M. Z. Kharadly and R. Ross, "Effect of wet antenna attenuation on propagation data statistics," IEEE Trans. Antennas Propag., vol. 49, no. 8, pp. 1183-1191, Aug. 2001, doi: 10.1109/8.943313.

[7] H. Leijnse, R. Uijlenhoet, and J. N. M. Stricker, "Microwave link rainfall estimation: Effects of link length and frequency, temporal sampling, power resolution, and wet antenna attenuation," Adv. Water Resour., vol. 31, no. 11, pp. 1481-1493, Nov. 2008, doi: 10.1016/j.advwatres.2008.03.004.

[8] A. Mancini, R. M. Lebron, and J. L. Salazar, "The Impact of a Wet $S$ Band Radome on Dual-Polarized Phased-Array Radar System
Performance," IEEE Trans. Antennas Propagat., vol. 67, no. 1, pp. 207220, Jan. 2019, doi: 10.1109/TAP.2018.2876733.

[9] C. Moroder, U. Siart, C. Chwala, and H. Kunstmann, "Modeling of Wet Antenna Attenuation for Precipitation Estimation From Microwave Links," IEEE Geosci. Remote Sens. Lett., pp. 1-5, 2019, doi: 10.1109/LGRS.2019.2922768.

[10] T. C. van Leth, A. Overeem, H. Leijnse, and R. Uijlenhoet, "A measurement campaign to assess sources of error in microwave link rainfall estimation," Atmospheric Meas. Tech., vol. 11, no. 8, pp. 46454669, Aug. 2018, doi: https://doi.org/10.5194/amt-11-4645-2018.

[11] J. Ostrometzky, R. Raich, L. Bao, J. Hansryd, and H. Messer, "The WetAntenna Effect-A Factor to be Considered in Future Communication Networks," IEEE Trans. Antennas Propagat., vol. 66, no. 1, pp. 315-322, Jan. 2018, doi: 10.1109/TAP.2017.2767620.

[12] C. Chwala et al., "Precipitation observation using microwave backhaul links in the alpine and pre-alpine region of Southern Germany," Hydrol. Earth Syst. Sci., vol. 16, no. 8, pp. 2647-2661, Aug. 2012, doi: 10.5194/hess-16-2647-2012.

[13] G. Smiatek, F. Keis, C. Chwala, B. Fersch, and H. Kunstmann, "Potential of commercial microwave link network derived rainfall for river runoff simulations," Environmental Research Letters, vol. 12, no. 3, p. 034026 , Mar. 2017, doi: 10.1088/1748-9326/aa5f46.

[14] A. Overeem, H. Leijnse, and R. Uijlenhoet, "Measuring urban rainfall using microwave links from commercial cellular communication networks," Water Resour. Res., vol. 47, no. 12, Dec. 2011, doi: 10.1029/2010WR010350.

[15] G. Roversi, P. P. Alberoni, A. Fornasiero, and F. Porcù, "Commercial microwave links as a tool for operational rainfall monitoring in Northern Italy," Atmos. Meas. Tech., vol. 13, no. 11, pp. 5779-5797, Oct. 2020, doi: 10.5194/amt-13-5779-2020.

[16] M. Fencl, M. Dohnal, P. Valtr, M. Grabner, and V. Bareš, "Atmospheric observations with E-band microwave links - challenges and opportunities," Atmos. Meas. Tech., vol. 13, no. 12, pp. 6559-6578, Dec. 2020, doi: 10.5194/amt-13-6559-2020.

[17] M. Fencl, P. Valtr, M. Kvičera, and V. Bareš, "Quantifying Wet Antenna Attenuation in $38-\mathrm{GHz}$ Commercial Microwave Links of Cellular Backhaul," IEEE Geoscience and Remote Sensing Letters, vol. 16, no. 4, pp. 514-518, 2019, doi: 10.1109/LGRS.2018.2876696.

[18] M. Graf, C. Chwala, J. Polz, and H. Kunstmann, "Rainfall estimation from a German-wide commercial microwave link network: optimized processing and validation for 1 year of data," Hydrol. Earth Syst. Sci., vol. 24, no. 6, pp. 2931-2950, Jun. 2020, doi: 10.5194/hess-24-29312020.

[19] M. F. Rios Gaona, A. Overeem, T. H. Raupach, H. Leijnse, and R. Uijlenhoet, "Rainfall retrieval with commercial microwave links in São Paulo, Brazil," Atmos. Meas. Tech., vol. 11, no. 7, pp. 4465-4476, Jul. 2018, doi: 10.5194/amt-11-4465-2018.

[20] M. Schleiss and A. Berne, "Identification of Dry and Rainy Periods Using Telecommunication Microwave Links," IEEE Geosci. Remote Sens. Lett., vol. 7, no. 3, pp. 611-615, Jul. 2010, doi: 10.1109/LGRS.2010.2043052.

[21] Specific attenuation model for rain for use in prediction methods, Rec. ITU-R P.838-3, International Telecommunication Union, 2005. [Online]. Available: http://www.itu.int/dms_pubrec/itu-r/rec/p/R-RECP.838-3-200503-I!!PDF-E.pdf

[22] Y. Xiang, S. Gubian, B. Suomela, and J. Hoeng, "Generalized Simulated Annealing for Efficient Global Optimization: the GenSA Package for R.," The R Journal Volume 5/1, June 2013, 2013, [Online]. Available: https://journal.r-project.org/archive/2013/RJ-2013-002/index.html.

[23] G. Villarini, P. V. Mandapaka, W. F. Krajewski, and R. J. Moore, "Rainfall and sampling uncertainties: A rain gauge perspective," J. Geophys. Res., vol. 113, no. D11, p. D11102, Jun. 2008, doi: 10.1029/2007JD009214. 